\title{
RADIO CONTINUUM AND RECOMBINATION LINE STUDIES OF THE GALACTIC CENTER
}

\author{
M. A. GORDON \\ National Radio Astronomy Observatory, ${ }^{*}$ Green Bank, W. Va., U.S.A.
}

\begin{abstract}
This review discusses unsuccessful searches for electromagnetic bursts accompanying gravitational events observed to come from the galactic center, radio observations of continuum emission and recombination lines from the region of the galactic center.
\end{abstract}

\section{Introduction}

In our attempts to understand the basic nature of our own Galaxy, perhaps the most important observations for us - and the most difficult - are the ones directed toward the galactic nucleus. The last few years have brought many new discoveries concerning the composition of the interstellar medium and the galactic center region. This paper presents a somewhat personal review of three areas of radio research which have been heavily pursued over the last few years.

\section{Pulsed E-M Radiation from the Galactic Center}

Weber (1969) has reported the detection of pulsed gravitational waves from the direction of the galactic center. His detectors have an angular resolution of $70^{\circ}$, so that uncertainty exists as to the precise direction from which the pulses originate. Subsequent papers establish that his detection rate is approximately two events per day and that the characteristic flux density of each event is approximately $3 \times 10^{28} \mathrm{Jy}$ at his observing frequency of $1660 \mathrm{~Hz}$.

The rationale for concomitant searches for pulsed electromagnetic (E-M) radiation is as follows. Presumably, the gravitational radiation arises from mass quadrupole moments associated with catastrophic events in the direction of the galactic center. Even if the coupling efficiency were extremely low, it seems reasonable either to expect these catastrophic events would also intrinsically generate bursts of detectable E-M radiation, or the energy released in the gravitational events would stimulate E-M bursts by some secondary process from material along the line of sight. The great sensitivities of apparatus designed to respond to E-M radiation could therefore be used to explore the nature of the gravitational events in an indirect manner. For example, even long-wave receivers can detect events of $100 \mathrm{Jy}$, a factor of $3 \times 10^{26}$ weaker than the gravitational bursts. Thus, the logic connecting pulsed gravitational and the expected E-M bursts is intuitive; it does not presume the detailed physical processes connecting the two events.

A large number of workers have searched for these associated E-M bursts at fre-

* Operated by Associated Universities, Inc., under contract with the National Science Foundation. 
quencies from $151.5 \mathrm{MHz}$ to (indirectly) beyond $5.6 \times 10^{12} \mathrm{MHz}$. When available, most workers have used receivers designed to reject terrestrial interference by requiring coincidence detection of the E-M pulses on either more than one frequency or at more than one location. To date, no E-M pulses have been found which correspond to the gravitational events recorded by Weber.

Figure 1 shows the observational limits of some of these searches, plotted as flux density in units of Jy against frequency in units of $\mathrm{Hz}$. Below $160 \mathrm{MHz}$ (Dulk, 1970; Brezgunov et al., 1971), the galactic center is undetectable presumably because of free-free absorption along the line of sight to the source; cross-hatching marks this region of the spectrum. These observational limits have a spectral index of -0.4 , but such an index probably only describes how instrumental sensitivities vary with frequency and does not describe a characteristic of the E-M bursts.

\section{LIMITS TO PULSED E-M RADIATION FROM GALACTIC CENTER}
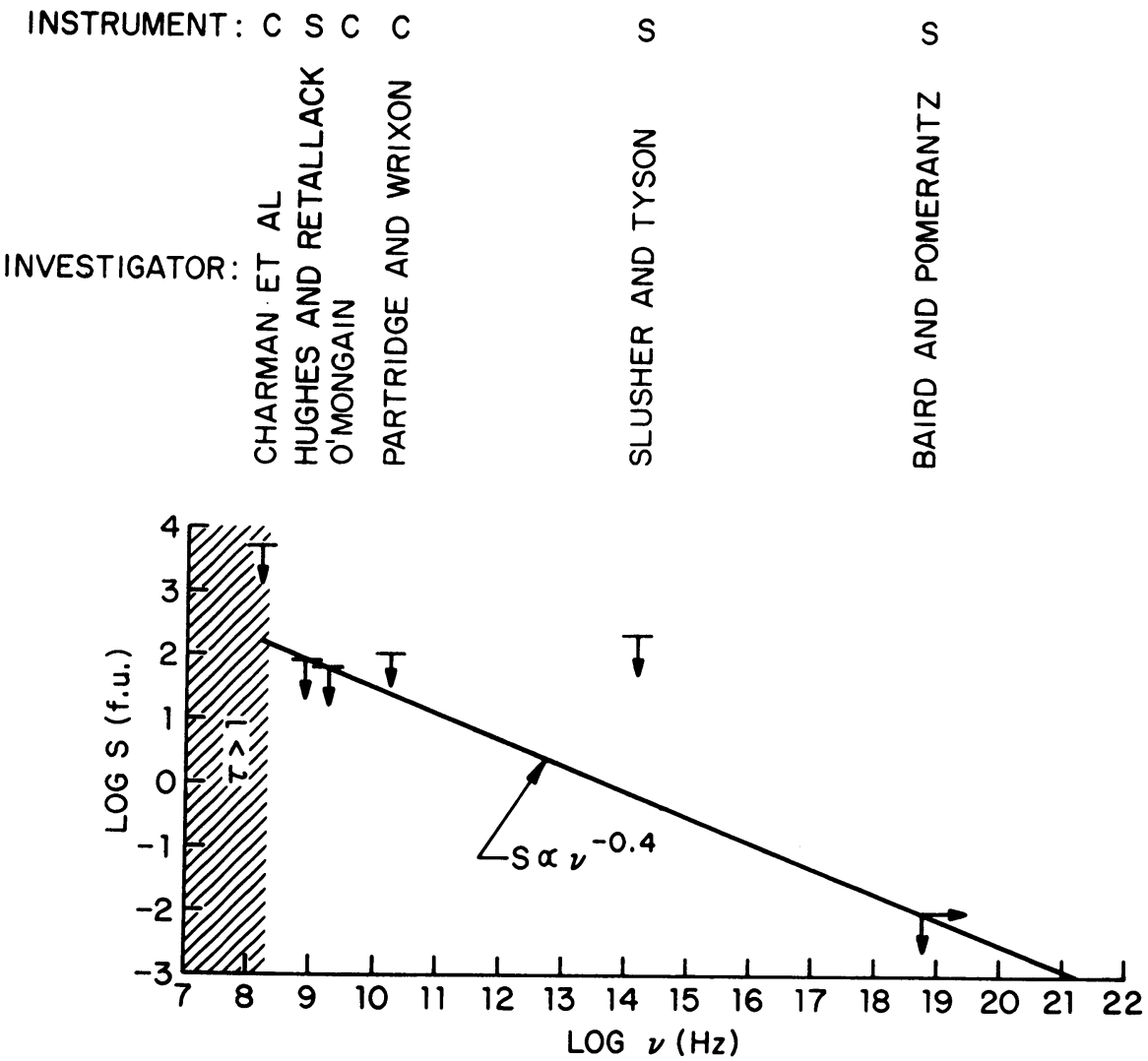

Fig. 1. Limits to electromagnetic pulses from the galactic center associated with observed gravitational events. The hatched region marks frequencies at which Sgr A cannot be detected. The fitted spectum probably describes instrumental sensitivities. Top: the investigator(s) for each observation is shown; $C$ or $S$ refers to a coincidence or single receiving system, respectively. 
Although no E-M pulses have been detected which correlate with the gravitational bursts, Hughes and Retallack (1973) do find discrete radio pulses of extra-terrestrial origin. Using a 60-ft telescope operating at $858 \mathrm{MHz}$, they find E-M pulses which cannot be identified with any radio, optical, infrared, X-ray, or $\gamma$-ray source within the beam during the drift scans across Sgr A, nor with solar or ionospheric events. Comparison observations made in other directions did not show the same kind of pulses. They conclude that the mysterious E-M pulses come from a region 4 min of time east of the galactic center but cannot identify their source.

The failure to find E-M pulses associated with Weber's gravitational events does not prove conclusively that they don't exist. Propagation of E-M pulses through ionized gas causes delays proportional to $v^{-2} N_{\mathrm{e}} \mathrm{d} l$; delays could be as large as $10^{\mathrm{m}}$ for pulses propagating at $150 \mathrm{MHz}$ through a column density of $10^{22}$ electrons $\mathrm{cm}^{-2}$, a reasonable estimate for the ionization between earth and Sgr A (see Partridge and Wrixon, 1972). Furthermore, the radio beams used in these searches usually are much smaller than the $70^{\circ}$ beam of Weber's apparatus; the source of the gravitational events may not be Sgr A. What is apparent, though, is that the flux density of E-M radiation associated with the gravitational pulses is an extremely small fraction $\left(<10^{-24}\right)$ of that radiated by the gravitational events.

\section{Maps of Continuum Emission}

Because of our location within the plane, absorption and scattering by interstellar gas and dust makes optical observations completely ineffective as a tool to study the nucleus of the Galaxy. This same interstellar matter also absorbs low-frequency radio waves, thereby limiting radio investigations to the centimeter and millimeter range. Furthermore, the high angular resolution required for these investigations can only be achieved (with circularly symmetric beams) by filled-aperture telescopes operated at short wavelengths; in this range, thermal sources necessarily predominate over nonthermal sources because of the intrinsic differences between the frequency dependences of the radio emission. Most interferometers are located in the northern hemisphere where synthesis of a symmetric beam to map the brightness distribution of Sgr A is extremely difficult owing to the low declination of the source; they usually produce strip scans either by name or effect. Lunar occultations also produce strip scans, but fortunately they frequently lie at considerable angles to one another according to the circumstances of each particular event. In short, the most important astronomical object within our Galaxy seems to be one of the most difficult to observe.

Figure 2 shows a recent map of the entire galactic center region made at $2 \mathrm{~cm}$ with a circularly symmetric beam of half-power width $2.25^{\prime}$ (Kapitsky and Dent, 1973). At a distance of $10 \mathrm{kpc}$, the map encloses a region $195 \times 270 \mathrm{pc}$. The region is complex, containing both thermal and nonthermal sources. The best known sources in the region are the $\mathrm{H}$ II region $\mathrm{Sgr}$ B2 and the central source $\mathrm{Sgr}$ A, whose positions are identified in the figure. There are also many smaller discrete sources seen in the region, some blended into the larger sources Sgr A and Sgr B. Maps of the same region, made 


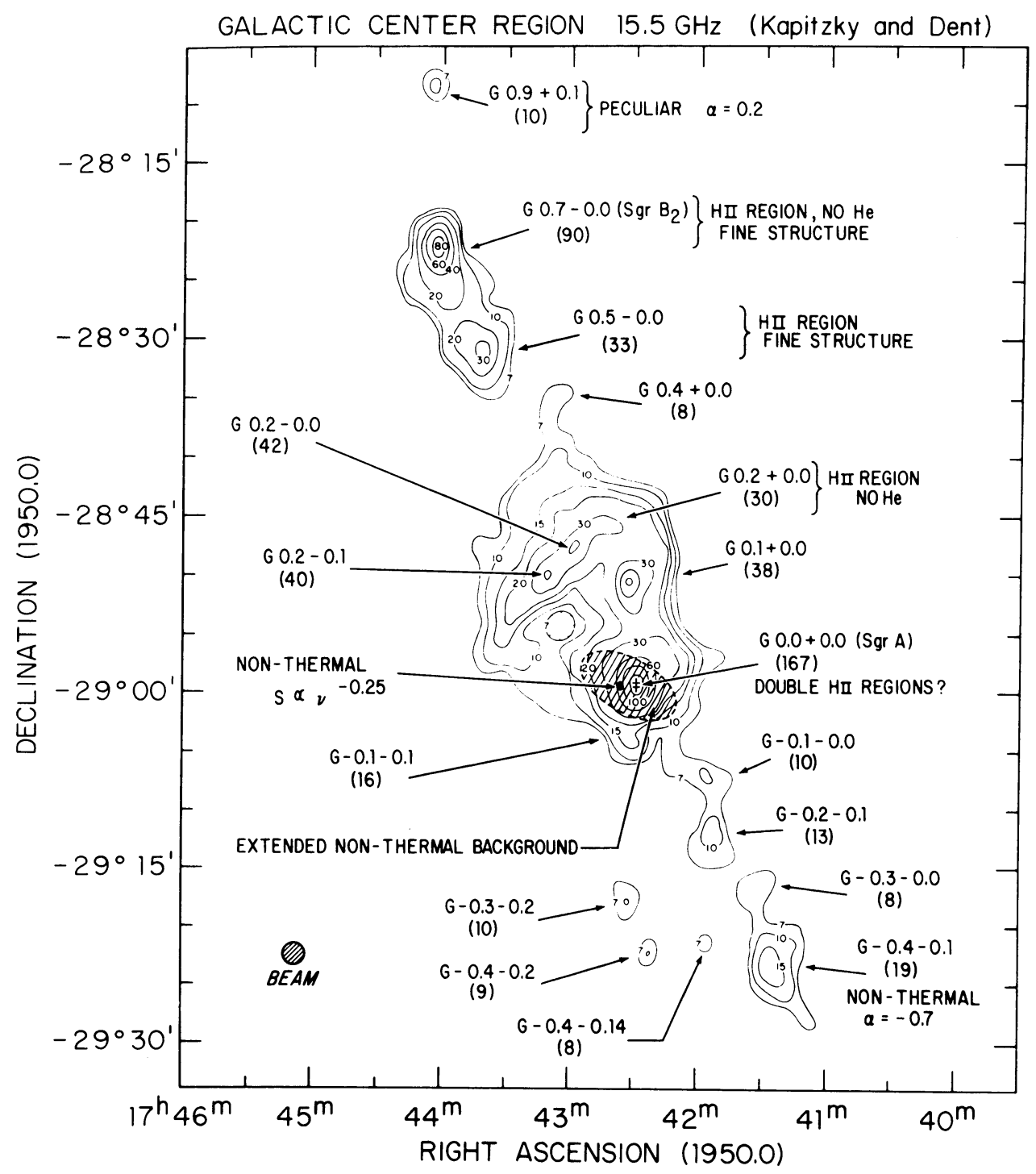

Fig. 2. The general region of the galactic center mapped at $2 \mathrm{~cm}$ with a beam of half-power width $2.25^{\prime}$ by Kapitsky and Dent (1973). Flux densities are shown by the numbers in parentheses.

at lower frequeneies (Whiteoak and Gardner, 1973), show the discrete sources to be superimposed upon an extended background several degrees in extent. An important result of the map shown in Figure 2 is that this background is no longer visible at $2 \mathrm{~cm}$; that is, this extended background must be nonthermal and any radio recombination lines seen against this background must arise in the $\mathrm{H}_{\mathrm{I}}$ gas along the line of sight.

From observations at $3.5 \mathrm{~mm}$, Hobbs et al. (1971) believe Sgr B2 to contain a dense, compact $\mathrm{H}$ II region. Martin and Downes (1972) interpret synthesis observations to 
show Sgr B2 to consist of seven discrete sources in an extended background. Recently synthesis observations by Balick and Sanders (1973) show Sgr B2 to have two discrete sources, less than 15 arcsec wide, containing 20 per cent of the flux at $13 \mathrm{~cm}$. Based upon the results of Hobbs et al., Balick and Sanders suggest these components have an electron density of $10^{6} \mathrm{~cm}^{-3}$ and an electron temperature of $15000 \mathrm{~K}$.

Sgr A, the radio source associated with the galactic center, is complex : it contains more than one component, and these components have different spectral indices. Evidently this complexity is the reason that, as has been known for some time, the overall source has been observed to shift in position and in angular size as a function of angular resolution and frequency. In the analysis of observations made at $327 \mathrm{MHz}$ by lunar occultation, and others at frequencies up to $1665 \mathrm{MHz}$, Gopal-Krishna et al. (1972) incorporated many previous observations - virtually strip scans - to produce the map of Sgr A shown in Figure 3.

Figure 3 shows the radio source $\operatorname{Sgr}$ A to contain two discrete peaks, $A$ and $S$, superimposed upon a broad background $B$ (not to be confused with $\mathrm{Sgr} B$ ) and another extended background $E$.

Component $A$ has a width of approximately $10^{\prime \prime}$ (Downes and Martin, 1971) and has the same position as the peak of the extended $2.2 \mu \mathrm{m}$ source seen by Becklin and Neugebauer (1969). On the basis of comparison of the ratio of the $2.2 \mu \mathrm{m}$ flux to star densities measured in the nucleus of M31, Sandage et al. (1969) and later Oort (1971) and Sanders and Lowinger (1972) all conclude that the infrared observations indicate that our Galaxy has a dense nucleus of approximately $10^{6} M_{\odot}$ in the form of population II stars within $1 \mathrm{pc}$ radius, the mass density of stars tapering off toward a radius of $\sim 750 \mathrm{pc}$ roughly as $R^{-1.8}$. Oort estimates the central star density (within a radius of $1.0 \mathrm{pc}$, or $\left.\sim 2.5^{\prime \prime}\right)$ to be approximately $10^{8}$ times that near the Sun. The radio data suggest these stars to be embedded in a nonthermal radiation field (component $A$ ) having a spectral index $\alpha$ of roughly $0.1 \pm 0.01$ between 327 and $5000 \mathrm{MHz}$.

Component $S$ is less well known because of blending with the extended background in the strip scans. Its position may be associated with that of the $40 \mathrm{~km} \mathrm{~s}^{-1}$ features of the $\mathrm{OH}$ and $\mathrm{H}_{2} \mathrm{CO}$ molecule (Sandqvist, 1971; Gardner and Whiteoak, 1972). Gopal-Krishna et al. suggest its spectral index is unlikely to be greater than 0.2 . (' $S$ ' indicates the name of its discoverer, Sandqvist.)

Components $B$ and $E$, because of their weaker intensities, are more difficult to classify as either thermal or non-thermal. They may be associated with the broad $100 \mu \mathrm{m}$ emission sources (Hoffman et al., 1971) tentatively associated with thermal emission from dust grains heated by the stars located near the galactic center (see Sanders and Lowinger [1972] for a general discussion). Gopal-Krishna et al. feel that the larger extended component $(E)$ has been missed in earlier work because of the great difficulty of observing it and its presumably steep spectral index. The spectral index of component $B$ appears to be no greater than 0.3. Both may be nonthermal, although by a similar analysis Lipovka (1971) reaches the opposite conclusion.

Figure 4 shows synthesis observations of Sgr A made by Balick and Sanders (1973) at $\lambda 13 \mathrm{~cm}$ with a beam $8^{\prime \prime} \times 20^{\prime \prime}$ and at $\lambda 4 \mathrm{~cm}$ with a beam $3^{\prime \prime} \times 7^{\prime \prime}$. The map shows two 


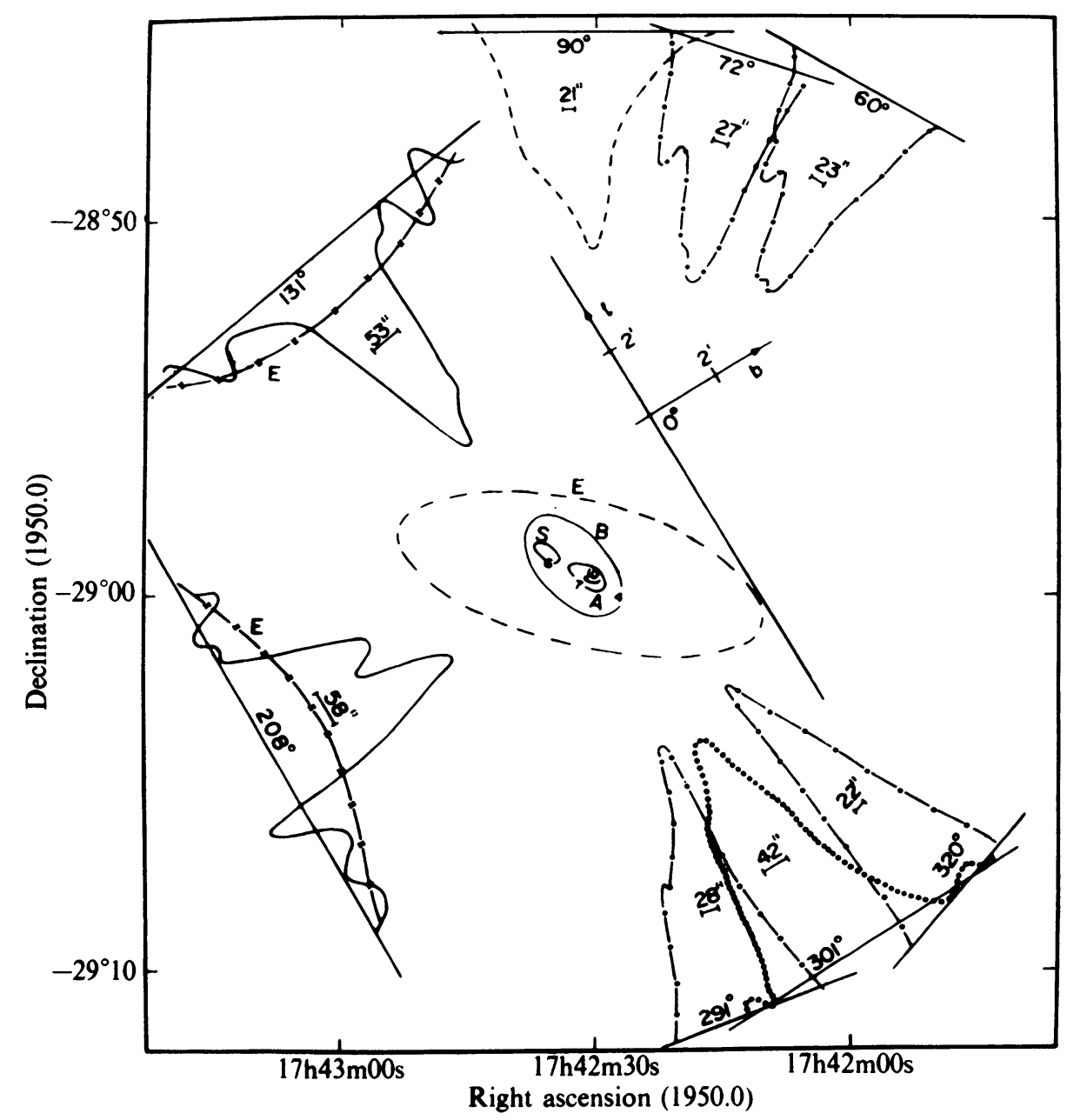

Fig. 3. A composite map of Sgr A made by Gopal-Krishna et al. (1972). In addition to their occultation observations made at $327 \mathrm{MHz}$ (solid lines), the map includes observations at $405 \mathrm{MHz}$ (dotted lines) by Thompson et al. (1969), at $1420 \mathrm{MHz}$ (dashed lines) by Sandqvist (1971).

distinct, unresolved sources containing approximately $20 \%$ of the total flux of $\mathrm{Sgr} \mathrm{A}$. The low-level background is nonthermal. These constitute the fine structure of the $A$ component of Figure 3. The $S$ component seen at low frequencies does not appear in these maps and hence is either nonthermal or too broad to appear in this synthesis observation. Note that the radio map shows the same double structure seen in the $10 \mu \mathrm{m}$ IR data.

The lowest frequency at which the galactic center region has been observed is $160 \mathrm{MHz}$. Figure 5 shows the map made at this frequency by Dulk and Slee (1973) with the Culgoora spectroheliograph. The peak emission comes from a position 




Fig. 4. The synthesis maps of fine structure in Sgr A made at $\lambda 13$ and $\lambda 4 \mathrm{~cm}$ by Balick and Sanders (1973). These maps have been 'cleaned'. Note the two discrete, unresolved sources superimposed upon a nonthermal background. The large cross marks the center of the extended source seen at $2.2 \mu \mathrm{m}$, the small cross the point source at $2.2 \mu \mathrm{m}$.

midway between components $A$ and $S$, and the broad component may well correspond to the source $E$ found at $327 \mathrm{MHz}$ by Gopal-Krishna et al. (1972).

At frequencies less than $120 \mathrm{MHz}$, the source Sgr A cannot be observed (Dulk, 1970; Brezgunov et al., 1971). The limits are consistent with free-free absorption along the line of sight such that the optical depth $\tau$ is 1 at approximately $200 \mathrm{MHz}$. As will be discussed below, this large-scale absorption is likely to be caused by partiallyionized cold gas in the interstellar medium.

\section{Radio Recombination Lines}

Radio recombination lines provide still another tool with which to investigate the galactic center. First, they are emitted only by thermal sources and can be used to classify sources of flat spectra as being either thermal or nonthermal. Second, the power emitted in the lines is a measure of $T_{\mathrm{e}}^{-1.5} \int n_{\mathrm{i}} n_{\mathrm{e}} \mathrm{d} l$ subject to the (often substantial) uncertainties imposed by departures from local thermodynamic equilibrium (LTE) and by large fluctuations in the physical conditions over the path length of the emission. Third, comparison of the frequency of the line center with the known rest frequency gives a radial velocity to a thermal source. Fourth, if the actual electron temperature can be established, the line widths are a measure of the nonthermal velocity fields (microturbulence) of the gas within the beam. Fifth, comparison of 


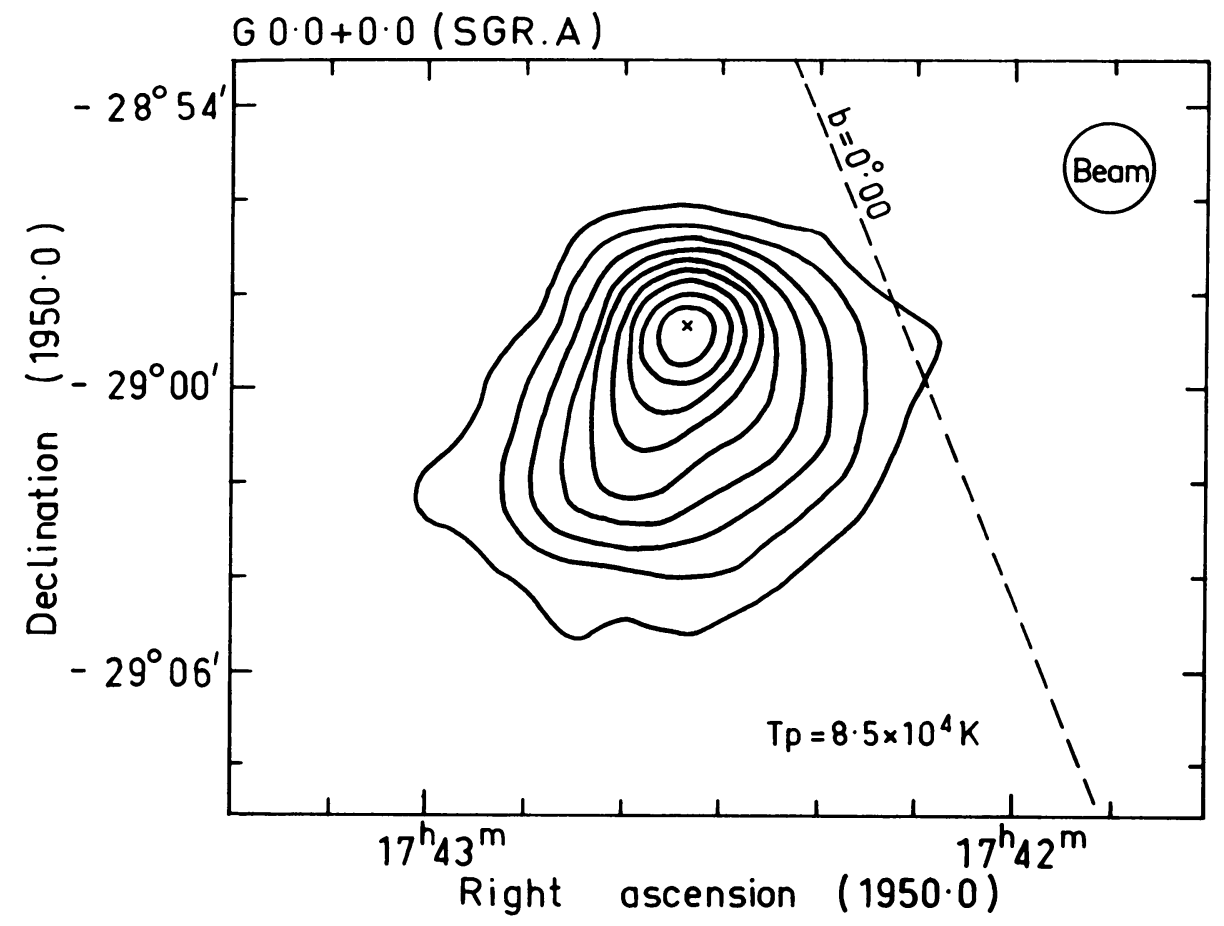

Fig. 5. Galactic center at $160 \mathrm{MHz}$ mapped by Dulk and Slee (1973).

lines emitted by different elements gives relative elemental abundances, also subject to the uncertainties due to departures of the level populations from LTE.

Surveys of radio recombination lines show that $\mathrm{H}$ II regions emit hydrogen recombination lines out of LTE (Brocklehurst and Seaton, 1972), at $5 \mathrm{GHz}$ have $\beta$ lines with intensities approximately $20 \%$ of the $\alpha$ lines (Hjellming and Gordon, 1971), and have helium lines with intensities $5 \%$ to $12 \%$ of the corresponding hydrogen lines (Churchwell and Mezger, 1973). Line profiles tend to be gaussian with full widths at half intensity of 20 to $30 \mathrm{~km} \mathrm{~s}^{-1}$, depending upon the relative angular sizes of the source and the beam (Sorochenko and Berulis, 1969; Smith and Weedman, 1970).

\section{(a) THE Sgr B2 COMPLEX}

Some sources located in the galactic center region (Figure 2) emit radio recombination lines. The giant Sgr B2 complex emits hydrogen lines having characteristics normal for $\mathrm{H}$ il regions. For example, the ratio of line to continuum intensities and the single measurement of the ratio of the $\alpha$ to $\beta$ line intensity are similar to those observed in the giant $\mathrm{H}_{\mathrm{II}}$ regions lying in the plane between galactic radii 3 and $10 \mathrm{kpc}$ (Mezger et al., 1972; Chaisson, 1973). On this basis, the G0.5-0.0 and Sgr B2 components have been identified as giant $\mathrm{H}$ II regions themselves. Mapping these 
components by means of recombination lines, Chaisson (1973) finds that the velocity gradients across these sources run in opposite directions, showing the G0.5-0.0 and Sgr B2 components to be physically separate.

Unlike most $\mathrm{H}$ II regions, the Sgr B complex does not emit detectable helium recombination lines. Huchtmeier and Batchelor (1973) report no detection of the He $109 \alpha$ line $(5 \mathrm{GHz})$ to a sensitivity of $1 \%$ of the corresponding hydrogen line. Rubin (1968) notes that a narrow range of late $O$ and early $B$ stars emit a radiation field capable of ionizing large amounts of hydrogen but very little helium; this situation is likely in the $\mathrm{H}$ II region Orion B (NGC 2024). However, the great amount of ionized hydrogen in the Sgr B2 complex requires on the order of $10^{2}$ early-type stars, and it is highly unlikely that all of these would lie in this narrow range of spectral types. If the Sgr B2 complex is an H II region, Churchwell and Mezger (1973) concluded that either the gas does not contain neutral or ionized helium, or that the ionization is not caused by early-type stars.

The detection of a large excess of $100-\mu \mathrm{m}$ flux may provide an alternative explanation for the deficiency of helium recombination lines in Sgr B2. Bless and Savage (1972) noted that, in general, the extinction by interstellar grains increases toward short wavelengths in the UV. Leibowitz (1973) used this fact to propose that, in H II regions showing no helium recombination lines, the grains deplete the radiation field shortward of $514 \AA$, reradiate the energy into the IR, and thereby prevent substantial ionization of helium within the $\mathrm{H}$ II region. Mezger et al. (1973) further explore the details of this mechanism. It seems possible that this mechanism can account for the lack of $\mathrm{He}$ II in the source Sgr B2.

Sgr B2 has some other unusual characteristics. Chaisson reports the widths of the recombination lines to be approximately $40 \mathrm{~km} \mathrm{~s}^{-1}$, much larger than those seen in other $\mathrm{H}$ II regions having similar ratios of angular size to beamwidth. Furthermore, Sgr B2 is an unusually rich source of molecules (see Gordon and Snyder, 1973). One component of the radial velocities of the molecules generally agrees with those of the recombination lines, suggesting the molecular cloud to be closely associated with the $\mathrm{H}$ II region. Chaisson (1973) suggests that the molecules lie within a dense shell of cold gas surrounding the $\mathrm{H}$ II region.

\section{(b) OTHER LINE SOURCES}

Regions closer to the galactic center also emit radio recombination lines. The extended region immediately to the north of Sgr A shows weak, frequently asymmetric hydrogen recombination lines, which Mezger et al. (1972) attribute to the ionized gas constituting this source. However, recombination lines are also seen in the direction of the nonthermal source Sgr A itself, and from the nonthermal extended region lying to the south of Sgr A (Lockman and Gordon, 1973). These spectra are similar to those of Sgr B2 in that no helium lines have been detected and that line widths are often large. Unlike those from $\mathrm{Sgr} \mathrm{B} 2$ the ration of the $\alpha$-to- $\beta$ line intensities is abnormally large for $\mathrm{H}$ II regions; that is, $\geqslant 9$ at $\lambda 18 \mathrm{~cm}$ (Lockman and Gordon, 1973) and $\geqslant 17$ at $\lambda 21 \mathrm{~cm}$ (Brown and Balick, 1973). Clearly, departures from LTE are extremely 
large for these recombination lines, much larger than that found for these lines in $\mathrm{H}$ II regions.

The line profile of the recombination lines seen in the direction of $\mathrm{Sgr} \mathrm{A}$ is highly non-gaussian. It contains a main component near $0 \mathrm{~km} \mathrm{~s}^{-1}$ which is seen in all other lines from the region and other components which vary from one beam position to another. The intensity of the main component correlates well with that of the background continuum. Furthermore, as Figure 6 shows, the line profile seen toward Sgr A bears a remarkable similarity to the corresponding absorption profile of $\mathrm{H}_{\mathrm{I}}$ at $\lambda 21 \mathrm{~cm}$, taken by an interferometer by Radhakrishnan et al. (1972).

Because of the great departures from LTE and the similarity of the line profile to that of $\mathrm{H} \mathrm{I}$ Lockman and Gordon (1973) considered that the $0 \mathrm{~km} \mathrm{~s}^{-1}$ feature of the recombination line, at least, might arise in the cold gas lying between $\mathrm{Sgr} \mathrm{A}$ and the Sun, stimulated by continuum radiation from the background sources according to the method described by Dupree and Goldberg (1969). Using the observational constraints and the non-LTE calculations of level populations (Dupree, 1972), they calculated that the lines could arise in dense, cold $(20 \mathrm{~K})$ clouds of a few parsecs in length, lying along the line of sight to Sgr A. The alternative hypothesis, that the lines arise in high temperature regions, requires the existence of long, small diameter 'fingers' of gas running from Sgr A directly to the Sun - a highly unlikely possibility.

The origin of these recombination lines in cold clouds also accounts for other observed phenomena. The clouds require electron densities $>3 \mathrm{~cm}^{-3}$, which would correspond to net ionization rates of $10^{-13} \mathrm{~s}^{-1}$ on the basis of ionization equilibrium. Similar rates are necessary to explain the weak recombination lines found in the plane in certain directions free of discrete radio sources (Gordon and Cato, 1972). Owing to pecularities associated with the unknown ionization mechanism and to the prob-

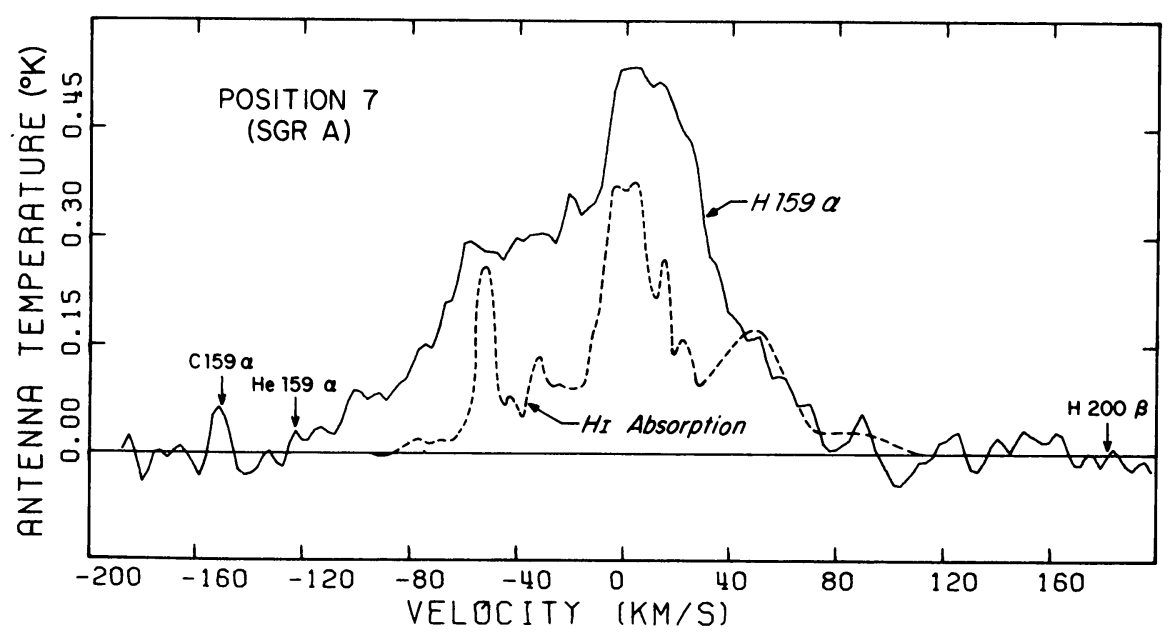

Fig. 6. Juxtaposition of the H159 $\alpha$-line profile (solid line) taken in the direction of Sgr A by Lockman and Gordon (1973) with an inverted absorption profile of neutral hydrogen taken in the same direction by Radhakrishnan et al. (1972). 
able effects of charge-transfer reactions, helium recombination lines might not arise (Brown and Balick, 1973). Furthermore, these clouds account for the impossibility of observing Sgr A at radio frequencies less than $200 \mathrm{MHz}$ because of free-free absorption (Dulk, 1970; Brezgunov et al., 1971). By independent observations and analysis, Brown and Balick (1973) reach the same conclusion, that the radio recombination lines seen at $\lambda 18 \mathrm{~cm}$ and $\lambda 21 \mathrm{~cm}$ in the general direction of Sgr A are emitted by dense, cold clouds along the line of sight to background sources.

The populations of levels involved in the $\lambda 3$ and $\lambda 6 \mathrm{~cm}$ lines observed by Mezger et al. (1972) may be substantially different than those of the apparently maser-prone levels involved in the $\lambda 18$ and $\lambda 21 \mathrm{~cm}$ lines. Therefore, the line intensities may differ greatly. The $\alpha$ lines seen at the shorter wavelengths involve levels near principal quantum numbers 85 and 109, where the atoms have effectively smaller collision cross-sections than at the more highly excited states seen at long wavelengths. For these levels, radiative processes may dominate so as to cause a uniform under-population and correspondingly weak recombination lines. For higher levels, the local collision and radiative processes populate in a way to make the levels highly sensitive to stimulation by background radiation. Therefore, it's possible that lines seen by Mezger et al. also come from the cold gas but are simply much weaker than those seen at lower frequencies. Clearly, additional observations of recombination lines, particularly of higher order lines, are necessary to understand the origin of the higher frequency lines from Sgr A.

Because helium recombination lines are not seen in Sgr B2 sources, it is tempting to consider the hydrogen lines seen there as arising from cold gas rather than to adopt the more radical explanations of elemental under-abundance of helium or strange new ionization mechanisms. Furthermore, the unusually wide lines observed by Chaisson (1973) could be a simple consequence of velocity dispersion (within the beam) over a long line of sight. The only impediment to such a model is the single observation of an apparently 'normal' $\beta$ line reported by Churchwell (1970). Because of its importance, this line should be reobserved, and other higher order lines be observed, so as to assess fully the details of the level populations of the hydrogen atoms before the Sgr B2 sources can be reliably classified as giant $\mathrm{H}$ II regions deficient in helium recombination lines. Alternatively, the details of the UV extinction of grains should be quantitatively explored.

\section{Summary}

The radio searches for electromagnetic radiation associated with bursts of graviational waves have been unsuccessful, in spite of truly enormous sensitivities. By themselves, these searches cast doubt on the validity of the reported graviational bursts; together with other unsuccessful direct searches for the gravitational bursts, the existence of the gravitational bursts seems unlikely at the fluxes quoted by Weber (1969).

Observations by both filled and synthetic apertures show the sources in the galactic center to be extremely complex, always having significant structure down to the resolution limit of the telescope. Such complexity makes it difficult to determine ac- 
curate spectral indices for these components, in some cases even to answer the simple question of whether the sources are thermal or nonthermal. Unfortunately, the detection (or not) of $\alpha$-type radio recombination lines does not discriminate between the two possibilities, because in principle these lines can arise in cold as well as hot gas. Lines of sight to the galactic center certainly pass through cold clouds of hydrogen, under circumstances where the amplification mechanism of Dupree and Goldberg (1969) is likely to be effective. Observations of higher order transitions can establish the nature of the level populations and hence the physical characteristics of the emitting gas.

\section{Acknowledgements}

It is a pleasure to thank Bruce Balick, Robert L. Brown, Ed Churchwell, Felix J. Lockman and Robert H. Sanders for many stimulating discussions on this subject.

\section{References}

Baird, G. A. and Pomerantz, M. A.: 1972, Phys. Rev. Letters 28, 1337.

Balick, B. and Sanders, R. H.: 1973, private communication.

Becklin, E. E. and Neugebauer, G.: 1969, Astrophys. J. Letters 157, L31.

Bless, R. C. and Savage, B. D.: 1972, Astrophys. J. 171, 293.

Brezgunov, V. N., Dagkesamansky, R. D., and Udaltsov, V. A.: 1971, Astrophys. Letters 9, 117.

Brocklehurst, M. and Seaton, M. J.: 1972, Monthly Notices Roy. Astron. Soc. 157, 179.

Brown, R. L. and Balick, B.: 1973, 'Observations of the Recombination Line Region Toward Sagittarius A', Astrophys. J., preprint.

Chaisson, E. J.: 1973, 'Microwave Spectroscopic Mapping of Gaseous Nebulae. IV: Excited Hydrogen in Sagittarius $\mathrm{B}_{2}$ ', Astrophys. J., preprint.

Charman, W. N., Fruin, J. H., Jelley, J. V., Haynes, R. F., Hodgson, E. R., Scott, P. F., Shakeshaft, J. R., Baird, G. A., Delaney, T. J., Lawless, B. G., Drever, R. W. P., and Meikle, W. P. S. : 1971, Nature 232, 177.

Churchwell, E.: 1970, 'Observations of Radio Recombination Lines of Hydrogen, Helium and Carbon', Indiana University, $\mathrm{Ph}$. D. thesis.

Churchwell, E. and Mezger, P. G.: 1973, Nature 242, 319.

Downes, D. and Martin, A. H. M.: 1971, Nature 233, 112.

Dulk, G. A.: 1970, Astrophys. Letters 7, 137.

Dulk, G. A. and Slee, O. B.: 1973, private communication.

Dupree, A. K.: 1972, Astrophys. J. 173, 293.

Dupree, A. K. and Goldberg, L.: 1969, Astrophys. J. Letters 158, L49.

Gardner, F. F. and Whiteoak, J. B.: 1972, Astrophys. Letters 10, 171

Gopal-Krishna, Swarup, G., Sarma, N. V. G., and Joshi, M. N.: 1972, Nature 239, 91.

Gordon, M. A. and Cato, T.: 1972, Astrophys. J. 176, 587.

Gordon, M. A. and Snyder, L. E.: 1973, Molecules in the Galactic Environment, John Wiley and Sons, New York.

Hjellming, R. M. and Gordon, M. A.: 1971, Astrophys. J. 164, 47.

Hobbs, R. W., Modali, S. B., and Maran, S. P.: 1971, Astrophys. J. Letters 165, L87.

Hoffman, W. F., Frederick, C. L., and Emery, R. J.: 1971, Astrophys. J. Letters 164, L23.

Huchtmeier, W. K. and Batchelor, R. A.: 1973, Nature 243, 155.

Hughes, V. A. and Retallack, D. S.: 1973, Nature 242, 105.

Kapitsky, J. E. and Dent, W. A. : 1973: 'A High Resolution Map of the Galactic Center Region', Astrophys. $J .$, preprint.

Leibowitz, E. M.: 1973, Astrophys. J. 181, 369.

Lipovka, N. M.: 1971, Astron. Zh. 48, 260 (English transl.: 1971, Soviet Astron. 15, 203.).

Lockman, F. J. and Gordon, M. A.: 1973, Astrophys. J. 182, 25. 
Martin, A. H. M. and Downes, D.: 1972, Astrophys. Letters 11, 219.

Mezger, P. G., Churchwell, E., and Pauls, T. A.: 1972, 'Ionized Gas in the Galactic Center' (to be published in the proceedings of the Regional Meeting of the IAU, Athens).

Mezger, P. G., Churchwell, E. B., and Smith, L. F.: 1973, 'IR-Excess Radiation and the Absorption Characteristics of Dust in Galactic Hil Regions', preprint.

O'Mongain, E.: 1973, Nature Phys. Sci. 242, 136.

Oort, J. H.: 1971, in D. J. K. O'Connell (ed.), Nuclei of Galaxies, Pontificiae Academiae Scientiarum Scripta Varia No. 35, North-Holland Publishing Co., Amsterdam, p. 11.

Partridge, R. B. and Wrixon, G. T.: 1972, Astrophys. J. Letters 173, L75.

Radhakrishnan, V., Goss, W. M., Murray, J. D., and Brooks, J. W.: 1972, Astrophys. J. Suppl. 4, 357.

Rubin, R.: 1968, Astrophys. J. 154, 391.

Sandage, A. R., Becklin, E. E., and Neugebauer, G.: 1969, Astrophys. J. 157, 55.

Sanders, R. H. and Lowinger, T.: 1972, Astron. J. 77, 292.

Sandqvist, A.: 1971, Ph.D. Thesis, University of Maryland.

Slusher, R. E. and Tyson, J. A.: 1973, Nature 243, 25.

Smith, M. G. and Weedman, D. W. : 1970, Astrophys. J. 160, 65.

Sorochenko, R. L. and Berulis, J. J.: 1969, Astrophys. Letters 4, 173.

Thompson, A. R., Riddle, A. C., and Lang, K. R.: 1969, Astrophys. Letters 3, 49.

Weber, J.: 1969, Phys. Rev. Letters 22, 1320.

Whiteoak, J. B. and Gardner, F. F.: 1973, Astrophys. Letters 13, 205.

\section{A. Gordon}

National Radio Astronomy Observatory,

2010 N. Forbes Ave., Tucson, Ariz. 85705, U.S.A.

\section{DISCUSSION}

Maxwell: Have the NRAO maps been laundered? That should be indicated on the maps.

Gordon: Yes, and I agree.

Robinson: Gordon has called for $\beta$ recombination line observations at Sgr B2. At Parkes we have measured the $\mathrm{H} 137 \beta$ line in Sgr B2. The line consists of two resolved components:

(i) A component centered on the H137 frequency $(5005.033 \mathrm{MHz}$ ) with similar width to the $\mathrm{H} 109 \alpha$ line and $20 \%$ of the $\mathrm{H} 109 \alpha$ intensity.

(ii) A component at higher frequency $\left(v_{0}=5005.320 \mathrm{MHz}\right)$ which is produced by the 3-3 A-branch transition in methanol! 